\title{
Der Mythos des ständigen Experten
}

\section{Philippe Ducor}

Rechtsanwalt, Professor an der Rechtsfakultät der Universität Genf; Arzt, Spezialist int. med.

\section{Kontext}

Im Jahr 2013 wurden innerhalb weniger Monate zwei junge Frauen - Marie und Adeline - von verurteilten Straftätern ermordet, denen eine Vollzugslockerung ihrer Strafen im Sinne von Art. 75a Abs. 2 des Strafgesetzbuches (StGB) gewährt worden war. Aufgrund dieser Ereignisse hat die Konferenz der Justiz- und Polizeidirektoren der lateinischen Schweiz (LKJPD) am 31. Oktober 2013 eine Empfehlung erarbeitet. Diese zielt darauf ab, dass die Kantone ihre Gesetzgebung dahingehend anpassen, dass Ärzte und andere Therapeuten hinsichtlich wichtiger Tatsachen betreffend die Gefährlichkeit der Täter gegenüber Behörden von der ärztlichen Schweigepflicht und dem Berufsgeheimnis entbunden werden. Die Kantone Genf, Waadt und Wallis haben sich entschieden, diese Empfehlung umzusetzen und sind dabei sogar über die Vorgaben der Empfehlung hinausgegangen. Die Gesetzesänderungen in den Kantonen sehen nicht bloss eine Lockerung der ärztlichen Schweigepflicht vor, sondern stipulieren eine echte Informationspflicht gegenüber den Behörden.

\section{Das aktuelle System}

Die aktuellen Bestimmungen betreffend die ärztliche Schweigepflicht sind in den Artikeln 321 und 17 des Strafgesetzbuches (StGB) geregelt. Gemäss Art. 321 Abs. 1 StGB macht sich ein Arzt strafbar, wenn er ein Geheimnis offenbart, welches ihm infolge seines Berufes anvertraut worden ist oder welches er in dessen Ausübung wahrgenommen hat. Diese Schweigepflicht ist allerdings nicht absolut. Gemäss Art. 321 Abs. 2 StGB ist der Arzt von seiner Schweigepflicht entbunden bzw. ist berechtigt auszusagen, wenn der Patient einwilligt oder die kantonalen Behörden auf Gesuch des Arztes hin die Aufhebung des Arztgeheimnisses schriftlich bewilligen. Zudem hält Art. 321 Abs. 2 StGB fest, dass die eidgenössischen und kantonalen Bestimmungen über die Zeugnispflicht und über die Auskunftspflicht gegenüber einer Behörde vorbehalten bleiben. ${ }^{1}$ Artikel 17 StGB enthält zudem einen Rechtfertigungsgrund, für den Fall einer unmittelbaren, nicht anders abwendbaren Gefahr eines Rechtsgutes (rechtfertigender Notstand). Wenn der Arzt die ärztliche Schweigepflicht gegenüber einem Inhaftierten verletzt, um ein Rechtsgut zu schützen - beispielsweise Leib und Leben eines Dritten - und dadurch ein höherwertiges Interesse geschützt wird, so handelt er rechtmässig und begeht keine Widerhandlung. Nach dem geltenden Recht sind die Gefängnisärzte in folgenden Fällen ermächtigt - nicht verpflichtet - sich über das Erfordernis der Einwilligung des Inhaftierten hinwegzusetzen und die Behörden zu informieren:

\section{Der Gefängnisarzt ist jedoch weder dafür} ausgebildet noch dazu qualifiziert, die Gefährlichkeit der Inhaftierten einzuschätzen.

- wenn sie auf Gesuch hin von der kantonalen Behörde von der ärztlichen Schweigepflicht entbunden worden sind. Dieser Weg ist jedoch nicht praktikabel, wenn die Gefahr unmittelbar ist;

- wenn ein rechtfertigender Notstand im Sinne von Art. 17 StGB vorliegt, d.h. wenn eine unmittelbare Gefahr besteht.

So hat der Arzt selbst die erforderliche Interessenabwägung vorzunehmen, anhand welcher er entscheidet, ob er sich mit einem Gesuch um Aufhebung des Arztgeheimnisses an die kantonale Behörde wendet oder ob er direkt die Behörden informiert. Eine solche Lösung ist insofern sachgerecht, als der Arzt selbst in die therapeutische Beziehung involviert ist und somit am besten in der Lage ist, die Situation zu bewerten. In der Praxis wendet sich der Gefängnisarzt entweder mit einem Gesuch um Aufhebung des Arztgeheimnisses an die kantonale Behörde oder er benachrichtigt die Behörden auf Anhieb, um so überwiegende Interessen, beispielsweise die öffentliche Sicherheit, zu wahren.

Mit anderen Worten, das heute geltende System funktioniert.

\section{Die geänderten Gesetze in der Westschweiz}

Die gesetzlichen Änderungen, die in den Kantonen Genf, Waadt und Wallis durchgeführt wurden, haben eines gemeinsam: Alle sehen eine Pflicht der Gefängnisärzte 


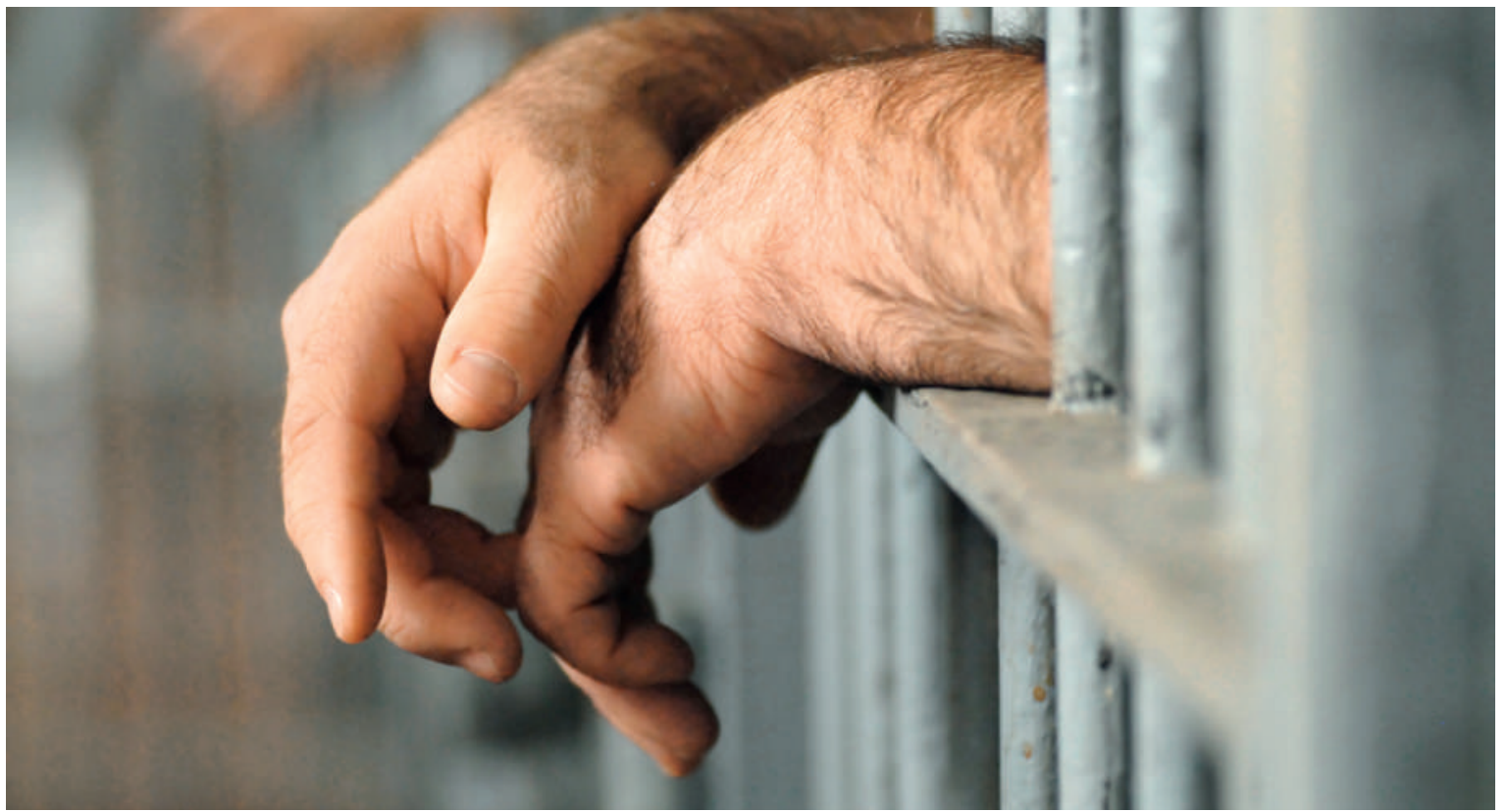

Gesetzesänderungen in den Kantonen Genf, Waadt und Wallis sehen eine Pflicht der Gefängnisärzte vor, den Inhaftierten, mit welchem sie in einer therapeutischen Beziehung stehen, unter gewissen Umständen bei der Behörde zu melden. So soll der Arzt die Behörden jederzeit über sämtliche wichtige Tatsachen betreffend die Gefährlichkeit des Inhaftierten benachrichtigen.

vor, den Inhaftierten, mit welchem sie in einer therapeutischen Beziehung stehen, unter gewissen Umständen bei der Behörde zu melden. Diese in den Gesetzen vorgesehenen Voraussetzungen der Anzeigepflicht entsprechen im Wesentlichen den in der Empfehlung der LKJPD vorgeschlagenen, d.h. wenn der Arzt Kenntnis über wesentliche Tatsachen betreffend die Gefährlichkeit des Inhaftierten erhält. Das erklärte Ziel dieser Empfehlung ist zwar lobenswert: die Verstärkung und Verbesserung der Kommunikation zwischen den Akteuren im Gefängnismilieu und jenen der öffentlichen Sicherheit. Jedoch wird der Entscheid, die Gefängnisärzte einer Anzeigepflicht zu unterstellen, leider die gegenteilige Wirkung haben.

\section{Zerstörung des Vertrauensverhältnisses}

Der Gefängnisarzt, welcher in einer therapeutischen Beziehung mit dem Inhaftierten steht, bleibt ein Arzt ("Äquivalenzgrundsatz»), unabhängig davon, ob diese Beziehung auf dem Willen des kranken Inhaftierten oder einer vom Gericht beschlossenen therapeutischen Massnahme basiert. Der Gefängnisarzt unterliegt somit der ärztlichen Schweigepflicht, ebenso wie der behandelnde Arzt, welcher auf dem öffentlichen Arbeitsmarkt tätig ist. Diese Vertraulichkeit ist die Grundlage für eine Vertrauensbeziehung, welche für eine gute Kommunikation und wirksame Behandlung notwendig ist. Die geänderten Gesetze zerstören dieses Vertrauensverhältnis, indem der Arzt zu einer Hilfsperson der Behörden gemacht wird. Es wird dem Inhaftierten, der sich einem Arzt anvertraut, unmöglich, zu bestimmen, welche Informationen vertraulich bleiben und welche an die Behörden weitergegeben werden, weil sie allenfalls als Indikator für seiner Gefährlichkeit angesehen werden können.

\section{Werden Inhaftierte nicht optimal therapiert, besteht beim Austritt ein erhöhtes Rückfall- risiko.}

\section{Gefahr für die öffentliche Sicherheit}

Die Zerstörung des Vertrauensverhältnisses, das für die therapeutische Beziehung unverzichtbar ist, wird einerseits die Wirksamkeit der Behandlung beeinträchtigen, vor allem im psychiatrischen Bereich. Andererseits wird dadurch bei den Inhaftierten Misstrauen geschürt. Werden Inhaftierte nicht optimal therapiert, besteht beim Austritt ein erhöhtes Rückfallrisiko. Künftig werden Inhaftierte zudem darauf verzichten, dem Arzt gewisse Dinge anzuvertrauen, die diesen bisher veranlassen konnten, die Behörden zu informieren. Eine weniger wirksame Behandlung und eine eingeschränkte Kommunikation tragen eindeutig nicht zur öffentlichen Sicherheit bei.

\section{Mythos des ständigen Experten}

Die geänderten Gesetze basieren auf einer gravierenden Verwechslung: Sie behandeln den Gefängnisarzt als «ständigen Experten», und laden ihm die Verantwortlichkeit auf, die Behörden jederzeit über sämtliche wich- 
tigen Tatsachen betreffend die Gefährlichkeit des Inhaftierten zu benachrichtigen. Der Gefängnisarzt ist jedoch weder dafür ausgebildet noch dazu qualifiziert, die Gefährlichkeit der Inhaftierten einzuschätzen. Auch wenn der Arzt im Rahmen des medizinischen Gefängnisdienstes oder einer vom Gericht beschlossenen therapeutischen Massnahme handelt, ist er vor allem

\section{Die geänderten Gesetze zerstören dieses Vertrauensverhältnis, indem der Arzt zu einer Hilfsperson der Behörden gemacht wird.}

ein behandelnder Arzt und nicht ein Experte zur Beurteilung der Gefährlichkeit. Das Strafgesetzbuch selbst betont indes die Unterscheidung zwischen behandelndem Arzt und einem Experten zur Beurteilung der Gefährlichkeit von Inhaftierten. Bei der Entscheidung über eine Lockerung des Straf- oder Massnahmenvollzuges (Urlaub, Arbeits- und Wohnexternat, bedingte Entlassung, usw.) (Art. 75a StGB), die Aufhebung einer Massnahme (Art. 62d Abs. 1 StGB) oder die Einführung einer therapeutischen Massnahme oder einer Internierung (Art. 56 Abs. 4 und $4^{\text {bis }}$ StGB) betreffend eine Person, die eine schwere Straftat im Sinne des Art. 64 Abs. 1 StGB $^{2}$ begangen hat, ist die Einholung eines Gutachtens erforderlich. Das Strafgesetzbuch hält fest: «Sachverständige und Vertreter der Psychiatrie dürfen den Täter nicht behandelt oder in anderer Weise betreut haben.» (Art. 62d Abs. 2 StGB) ${ }^{3}$.

\section{Wieso lediglich Gesundheitsfachleute?}

Art. 321 StGB ist dem Berufsgeheimnis gewidmet und gilt nicht nur für Gesundheitsfachleute, sondern auch für Anwälte und Seelsorger, von welchen zahlreiche im Gefängnismilieu tätig sind. Solange die der Anzeigepflicht unterliegenden Gefängnisärzte weder fachgerecht ausgebildet noch qualifiziert sind, um die Gefährlichkeit der Inhaftierten einzuschätzen, werden Informationen, die sie betreffend die Gefährlichkeit erhalten, nicht spezifischer sein als solche, von denen Anwälte und Seelsorger Kenntnis erlangen könnten. Es ist daher erstaunlich, dass die geänderten Gesetze lediglich die Gesundheitsfachleute im Gefängnismilieu betreffen und nicht auch anderen Berufe, welche unter Art. 321 StGB fallen. Den Politikern, worunter zahlreiche Anwälte sind, war offenbar klar, dass Anwälte ihren Beruf nicht fachgemäss ausüben könnten, wenn sie betreffend ihren Klienten einer solchen Anzeigepflicht unterliegen würden. In diesem Zusammenhang ist es zu bedauern, dass dieselbe Überlegung hinsichtlich der Gesundheitsfachleute nicht berücksichtigt wurde.

\section{Übertragung der Verantwortung von den Behörden auf den Arzt}

Die meisten Inhaftierten kommen während ihres Freiheitsentzugs regelmässig in Kontakt mit einem Gefängnisarzt. Der einer Anzeigepflicht unterstehende Gefängnisarzt wird in Fällen, in welchen ein Inhaftierter erneut eine Straftat begeht - nachdem eine Lockerung des Strafvollzuges im Sinne von Art. 75a Abs. 2 StGB gewährt wurde oder die Massnahme im Sinne von Art. 75a Abs. 2 StGB aufgehoben wurde - schnell zum Sündenbock werden. Im Endeffekt ist es ein Leichtes, dem Gefängnisarzt a posteriori vorzuwerfen, er habe es versäumt, den Behörden die notwendigen Informationen betreffend die Gefährlichkeit des Inhaftierten mitzuteilen und diese Gefahr habe sich nun realisiert. Diese Übertragung der Verantwortung der Behörden auf die Gesundheitsfach-

Entgegen ihrem erklärten Ziel, wirken die geänderten Gesetze damit zum Nachteil der öffentlichen Sicherheit.

leute mutet seltsam an, wenn man an das Drama zurückdenkt, welches Adeline das Leben gekostet hat, und bedenkt, dass es dort die Behörden unterlassen haben, ein Gutachten - welches vorgesehen war - betreffend die Gefährlichkeit des Mörders zu erstellen.

\section{Schlussfolgerung}

Die geänderten Einführungsgesetze des Strafgesetzbuches in den Kantonen Genf, Waadt und Wallis sind eine "schlechte gute Idee» und verstossen gegen das Grundprinzip der ärztlichen Schweigepflicht. Indem das erforderliche Vertrauensverhältnis, welches für jede therapeutische Beziehung unverzichtbar ist, zerstört wird, wirkt die Gesetzesänderung dem erklärten Ziel der öffentlichen Sicherheit entgegen. In Genf lancierte die Association des Médecins (AMG) am 4. März 2016 eine Initiative in Form eines ausgearbeiteten Entwurfs auf kantonaler Ebene. Diese übernimmt im Wesentlichen den Text des in Genf geänderten Gesetzes, um so die begrüssenswerte Zielsetzung beizubehalten, jedoch unter gleichzeitiger Wiederaufnahme des Grundprinzips der ärztlichen Schweigepflicht. Der Wortlaut der Initiative bestätigt das Recht des Arztes, unter qualifizierten Umständen die Behörden zu benachrichtigen, schliesst jedoch jegliche Anzeigepflicht zu seinen Lasten aus.

Bildnachweis

Willeecole | Dreamstime.com 